\title{
Eficiência no uso da água de duas cultivares de batata-doce em resposta a diferentes lâminas de irrigação
}

\author{
Everardo C Mantovani'; Fábio T Delazari'; Luiz Eduardo Dias²; Igor R de Assis ${ }^{2}$; Gustavo HS Vieira ${ }^{3}$; \\ Felipe M Landim ${ }^{1}$ \\ ${ }^{1} U F V$, Depto de Eng. Agrícola, 36570-000 Viçosa-MG; everardo@ufv.br, fabio.delazari@ufv.br; felipe.landim@ufv.br; ${ }^{2} U F V$, Depto de \\ Solos; ledias@ufv.br; igor.assis@ufv.br; ${ }^{3}$ IFES-ES' Diretoria de Administração do campus Centro Serrano, Rod. ES 010, Manguinhos, \\ 29173-087 Serra-ES; ghsvieira@gmail.com
}

\section{RESUMO}

A crescente preocupação mundial com a produção eficiente de energia e com os recursos hídricos exige desenvolver estratégias que economizem água sem redução da produtividade. Assim, objetivou-se determinar as lâminas de irrigação que resultem na máxima produtividade e uso eficiente de água para duas cultivares de batata-doce. Lâminas equivalentes a 50, 75, 100 e $125 \%$ da evapotranspiração da cultura (ETc), calculadas por meio do software Irriplus, foram aplicadas via gotejamento. A produtividade de raízes tuberosas foi determinada após 187 e 208 dias de cultivo das cultivares de batata-doce Amanda e Duda, respectivamente. A maior produtividade (49,8 $\mathrm{t} \mathrm{ha}^{-1}$ ) de raízes tuberosas pelas plantas da 'Amanda' foi obtida com uma lâmina acumulada de 325,5 mm (95,2\% da ETc), enquanto que para 'Duda' a lâmina que proporcionou maior produtividade $(67,1$ $\left.\mathrm{t} \mathrm{ha}^{-1}\right)$ foi de $347,0 \mathrm{~mm}(100,4 \%$ da ETc $)$. A diferença de produtividade entre as cultivares foi de $25,8 \%$, com aumento no consumo de água pela cultivar Duda de $21,5 \mathrm{~mm}(6,2 \%$ maior). Isso indica que 'Duda' apresentou maior eficiência no uso da água. O aumento na lâmina de água aplicada resultou no aumento da eficiência no uso da água (EUA), até atingir um valor máximo de $16,1 \mathrm{~kg} \mathrm{~m}^{-3}$, com a aplicação de 301,8 mm (87,3\% da ETc) para 'Amanda' e de 20,0 kg $\mathrm{m}^{-3}$, com a aplicação de $332,4 \mathrm{~mm}(96,2 \%$ da ETc) para 'Duda'. A produtividade referente à máxima EUA foi de 48,6 $\mathrm{tha}^{-1}$, com uma economia de $23,7 \mathrm{~mm}$, representando redução de apenas $1,2 \mathrm{t} \mathrm{ha}^{-1}$ na produtividade da cultivar Amanda. Para 'Duda', a produtividade equivalente à máxima EUA foi de $66,3 \mathrm{t} \mathrm{ha}^{-1}$, com uma economia de $14,6 \mathrm{~mm}$ e redução de $0,8 \mathrm{t} \mathrm{ha}^{-1}$, quando comparada à produtividade real. As lâminas recomendadas para 'Amanda' e 'Duda', nas condições edafoclimáticas de condução desta pesquisa, foram de 301,8 e $332,4 \mathrm{~mm}$, respectivamente.

Palavras-chave: Ipomoea batatas, manejo de irrigação, economia de água.

\begin{abstract}
Yield and water use efficiency for two sweet potato cultivars depending on irrigation depths

The increasing global concern with the efficient production of energy and water resources requires the development of strategies that save water without reducing yield. Therefore, this study was carried out to determine the irrigation depth that provides maximum yield and water efficient use for two sweet potato cultivars. Irrigation depths equivalent to $50,75,100$ and $125 \%$ of the crop evapotranspiration (ETc), calculated by the Irriplus software, were evaluated using a drip irrigation system. The tuberous root yield was determined 187 and 208 days after planting date for Amanda and Duda cultivars, respectively. The highest productivity $\left(49.8 \mathrm{t} \mathrm{ha}^{-1}\right)$ of the tuberous roots for 'Amanda' was obtained with a cumulative irrigation depth of $325.5 \mathrm{~mm}$ ( $95.2 \%$ of ETc), while for 'Duda' the irrigation depth that resulted in highest productivity $\left(67.1 \mathrm{t} \mathrm{ha}^{-1}\right)$ was $347.0 \mathrm{~mm}$ (100.4\% of ETc). The productivity difference between cultivars was $25.8 \%$, presenting increase in water consumption by 'Duda' of 21.5 $\mathrm{mm}$ (6.2\% higher). This comparison indicates that 'Duda' presents higher water use efficiency. The increasing irrigation depth resulted in the highest water efficient use (EUA), with maximum value of 16.1 $\mathrm{kg} \mathrm{m}^{-3}$, for irrigation depth of $301.8 \mathrm{~mm}(87.3 \% \mathrm{ETc})$ for 'Amanda'. For 'Duda' the EUA was $320.0 \mathrm{~kg} \mathrm{~m}^{-3}$, with irrigation depth of 332.4 $\mathrm{mm}(96.2 \% \mathrm{ETc})$. The productivity obtained with the maximum EUA was $48.6 \mathrm{t} \mathrm{ha}^{-1}$, representing economy of $23.7 \mathrm{~mm}$, or productivity reduction of only $1.2 \mathrm{t} \mathrm{ha}^{-1}$ for 'Amanda'. 'Duda' showed productivity equivalent to maximum EUA of $66.3 \mathrm{tha}^{-1}$, representing economy of $14.6 \mathrm{~mm}$, or productivity reduction of $0.8 \mathrm{tha}^{-1}$. The irrigation depths recommended for 'Amanda' and 'Duda', in present edaphoclimatic conditions, were 301.8 and $332.4 \mathrm{~mm}$, respectively.
\end{abstract}

Keywords: Ipomoea batatas, irrigation management, water economy.

\section{(Recebido para publicação em 29 de janeiro de 2013; aceito em 18 de novembro de 2013)}

(Received on January 29, 2013; accepted on November 18, 2013)

$\mathrm{D}^{\mathrm{s}}$ evido ao decréscimo de disponibilidade de água para a agricultura, aos altos custos de energia (López-Mata et al., 2010) e a crescente preocupação mundial com os recursos hídricos, torna-se necessária a adoção de estratégias de manejo que possibilitem economia de água sem prejuízos de produtividade das culturas que demandam irrigação. Uma boa estratégia de manejo da irrigação é fundamental para economizar água sem, no entanto, por em risco o rendimento das culturas (Jalota et al., 2006; Pereira et al., 2009).
O manejo adequado e estratégico da água pode ser feito utilizando-se o índice de eficiência de uso da água para o planejamento e a tomada de decisão da irrigação, aumentando-se assim, a produtividade das culturas (Karatas et $a l ., 2009)$. O uso de informações rela- 
cionadas à produtividade e à eficiência do uso da água são importantes para a análise econômica do sistema produtivo, além de auxiliar na economia do uso da água (Sandri et al., 2007).

A eficiência do uso da água (EUA) relaciona a produção de biomassa ou produção comercial pela quantidade de água aplicada ou evapotranspirada. Em agricultura irrigada, a elevação e a determinação dos níveis desta eficiência são bastante complexos e requerem conhecimentos e considerações interdisciplinares; todavia, Dinar (1993) menciona que existem meios para se elevar os valores da eficiência do uso da água destacando-se entre esses, o manejo adequado de irrigação. Assim, as pesquisas procuram objetivar altos valores da EUA, mantendo-se altas produtividades (Buzetti et al., 1993).

A batata-doce (Ipomoea batatas) é uma espécie pertencente à família Convolvulaceae originária da América do Sul, e possui uma ampla adaptação às condições climáticas do Brasil. Atualmente, ocupa a sexta posição entre as olerícolas mais consumidas no Brasil (Silva et al., 2008), além de possuir grande importância sócio-econômica participando no suprimento de calorias, vitaminas e minerais na alimentação humana (Oliveira et al., 2005).

É considerada uma cultura rústica, pois apresenta grande resistência a pragas, boa resposta à aplicação de fertilizantes, desenvolvendo-se em solos de baixa fertilidade e até degradados. Além disso, apresenta tolerância ao déficit hídrico, custo de produção relativamente baixo, com investimentos mínimos e retorno elevado (Pereira Junior et al., 2008). Devido à sua rusticidade, poucas são as pesquisas realizadas com esta cultura no Brasil, principalmente no que se refere à demanda hídrica.

Segundo Doorenbos \& Kassam (1979), a produtividade de uma cultura é função de complexos processos biológicos, fisiológicos, físicos e químicos, os quais são determinados pelas condições ambientais (clima, solo e água) e por fatores genéticos. Neste sentido, novas cultivares vêm sendo desenvolvidas a fim de se obter plantas de elevada produtividade, que possuem raízes tuberosas com maiores concentrações de amido, visando o uso energético da cultura para a produção de etanol (Dias, 2010).

A fotossíntese das plantas é limitada pela restrição da abertura estomática, em condição de déficit hídrico (Yordanov et al., 2003). Esse processo fisiológico vital para as plantas é então comprometido em condição de baixa disponibilidade de água, ocasionando decréscimos na produção de carboidratos que posteriormente seriam armazenados (Singels et al., 2005).

Devido à crescente preocupação com os recursos hídricos, este trabalho teve como objetivo determinar as lâminas de irrigação que possibilitam a máxima produtividade e a máxima eficiência no uso da água por duas cultivares de batata-doce recomendadas para produção de etanol, nas condições edafoclimáticas de Viçosa-MG.

\section{MATERIAL E MÉTODOS}

O experimento foi instalado em casa de vegetação do Departamento de Engenharia Agrícola da UFV, Viçosa-MG $\left(20^{\circ} 45^{\prime} 14^{\prime \prime} \mathrm{S}, 42^{\circ} 52^{\prime} 55^{\prime}\right.$ 'O, altitude $648 \mathrm{~m}$ ). As cultivares utilizadas foram Amanda e Duda, obtidas pelo programa de melhoramento genético desenvolvido pela Universidade Federal do Tocantins.

O solo na área experimental foi classificado como argiloso, apresentando $\mathrm{pH}$ de 6,1 , saturação por bases igual a $75 \%$ e teor médio de matéria orgânica igual a 2,4 dag $\mathrm{kg}^{-1}$. O valor médio de densidade do solo foi de $1,30 \mathrm{~g} \mathrm{~cm}^{-3}$ e os valores de umidade volumétrica referentes à capacidade de campo e ponto de murcha permanente foram de 0,384 e $0,218 \mathrm{~m}^{3}$. O preparo do solo para o plantio foi realizado com enxada rotativa, seguida da formação manual das leiras espaçadas de 1,0 m. A análise química do solo realizada previamente ao plantio demonstrou que o mesmo possuía condição de eutrofismo, com $\mathrm{pH}=6,1$; teores disponíveis de $\mathrm{P}$ e $\mathrm{K}$ de 58,1 e $557,0 \mathrm{mg} \mathrm{dm}^{-3}$ respectivamente;

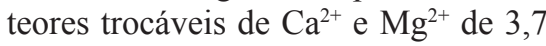
e 1,2 $\mathrm{cmol}_{\mathrm{c}} \mathrm{dm}^{-3}$, respectivamente; e saturação de bases igual a 75,2\%, portanto não sendo necessárias práticas de correção da acidez e adubações com fertilizantes fosfatados e potássicos. Foi realizada apenas adubação nitroge- nada (200 $\mathrm{kg} \mathrm{ha}^{-1}$ de N) (Oliveira et al., 2006), dividida em duas aplicações, aos 30 e 60 dias após o plantio com sulfato de amônio. A injeção do fertilizante no sistema de irrigação foi feita com o auxílio do venturi.

A cultura foi irrigada por gotejamento, com vazão real de $1,71 \mathrm{~L} \mathrm{~h}^{-1} \mathrm{e}$ espaçamento entre gotejadores de 30 $\mathrm{cm}$. O sistema apresentou Coeficiente de Uniformidade de Christiansen (CUC) de $94 \%$ e intensidade de aplicação de $5,70 \mathrm{~mm} \mathrm{~h}^{-1}$.

Com auxílio do software Irriplus (2008) determinou-se a demanda hídrica da batata-doce, utilizando-se coeficientes de ajuste sobre a evapotranspiração de referência (ETo). A lâmina bruta de irrigação foi calculada por meio de um balanço hídrico, em que a entrada de água foi a irrigação e a saída, a evapotranspiração da cultura (ETc). Utilizou-se a Equação 1, proposta por Doorenbos \& Pruitt (1977), modificada por Mantovani \& Costa (1998), para se estimar a evapotranspiração da cultura:

\section{$E T c=E T o \times K c \times K s \times K l$ \\ (equação 1)}

em que $E T c=$ evapotranspiração da cultura, em $\mathrm{mm} \mathrm{d}^{-1} ; E T o=$ evapotranspiração de referência, em $\mathrm{mm} \mathrm{d}^{-1} ; K c=$ coeficiente de cultura adimensional; $K s=$ coeficiente de estresse hídrico adimensional e, $K l=$ coeficiente de localização adimensional.

O método de estimativa da ETo utilizado pelo Irriplus (2008), de acordo com os elementos meteorológicos disponíveis (radiação solar, temperatura média, velocidade do vento, umidade relativa) foi o modelo de Penman-Monteith-FAO (Allen et al., 1998). Os dados meteorológicos utilizados para a realização do experimento foram obtidos em uma estação agrometeorológica automática localizada na casa de vegetação.

O valor do coeficiente de estresse (Ks) foi calculado utilizando-se o modelo logarítmico proposto por Bernardo et al. (2006), empregando a equação 2 :

$$
K s=\frac{\operatorname{In}(L A A+1)}{\operatorname{In}(C T A+1)} \text { (equação 2) }
$$

em que, $K S=$ coeficiente de estresse; $L A A=$ lâmina atual de água no solo, em 
$\mathrm{mm} ; C T A=$ capacitada total de água no solo, em mm.

O valor do coeficiente de localização de irrigação $(\mathrm{Kl})$ foi calculado em função da porcentagem de área molhada ou sombreada, utilizando-se o modelo proposto por Keller \& Bliesner (1990), conforme a equação:

$$
K l=0,1 \sqrt{P} \quad(\text { equação } 3)
$$

em que, $K l=$ coeficiente de localização de irrigação, adimensional; $P=$ porcentagem de área molhada ou sombreada.

Foram utilizados valores do coeficiente de cultura $(\mathrm{Kc})$, porcentagem de área sombreada/molhada (P), profundidade efetiva do sistema radicular e o fator de disponibilidade de água (Tabela 1).

Os valores de Kc considerados neste experimento estão próximos aos valores obtidos em cultivos de batata-doce em Moçambique, onde foram determinados os valores de 0,55-0,70; 1,1-1,2 e 0,8 para as fases de estabelecimento, intermediária e final de ciclo, respectivamente (Gomes \& Carr, 2003).

As lâminas brutas foram calculadas pelo somatório das ETc no intervalo entre irrigações (três dias), indicando a necessidade hídrica da cultura, considerando a uniformidade de aplicação do sistema e a área adequadamente irrigada de $80 \%$.

A umidade do solo foi monitorada pelo método termogravimétrico (EMBRAPA, 1997), com amostragens mensais, utilizando trado holandês, de modo a permitir o acompanhamento do balanço hídrico do solo que, comparado ao consumo de água pela cultura, estimado pelo aplicativo Irriplus (2008), garantiu a aferição do método.

O delineamento experimental utilizado foi em blocos casualizados, com quatro tratamentos e quatro repetições, sendo cada parcela experimental composta por 18 plantas com espaçamentos de 0,25 m entre plantas e 1,0 m entre linhas. A parcela útil foi composta por quatro plantas por repetição. Os tratamentos 1, 2, 3 e 4 referem-se a lâminas de 50, 75, 100 e 125\% da ETc, respectivamente, correspondendo às lâminas de 217,$8 ; 281,8 ; 345,7$ e 409,6 mm para a cultivar Amanda e de 246,4; 311,$1 ; 375,9$ e 440,6 mm para a cultivar Duda. Estas lâminas foram aplicadas até os 187 e 208 dias após o plantio (final do experimento) para 'Amanda' e 'Duda', respectivamente.

Para determinação da produtividade de raízes tuberosas foi realizada colheita manual após 187 e 208 dias de cultivo da 'Amanda' e 'Duda', respectivamente, períodos estes sugeridos como final do ciclo de cada cultivar em função da maior produção de etanol (Silveira, 2007). As raízes tuberosas mais representativas de quatro plantas, em cada parcela, foram utilizadas para a estimativa da produtividade A eficiência no uso da água (EUA) no final do ciclo fenológico foi definida pela relação entre a produção $(\mathrm{P})$ e a quantidade de água aplicada (L), expressa em $\mathrm{kg} \mathrm{m}^{-3}$. A equação seguinte foi utilizada no cálculo do EUA:

$E U A=\frac{P}{L} \quad$ (equação 4)

em que, $E U A=$ uso eficiente da água, $\mathrm{kg} \mathrm{m}{ }^{-3} ; P=$ produção, $\mathrm{kg}$; =- lâmina aplicada, $\mathrm{m}^{3}$.

Os dados obtidos foram submetidos às análises de variância e regressão, utilizando o programa estatístico SAEG 9.1 (SAEG, 2007). Os coeficientes encontrados para as equações de regressão foram submetidos ao teste " $\mathrm{t}$ ", com posterior correção, considerando-se o quadrado médio do resíduo, os graus de liberdade da ANOVA e o quadrado médio independente da regressão.

\section{RESULTADOS E DISCUSSÃO}

O aumento da lâmina d'água aplicada resultou em aumento da produtividade de raízes tuberosas de ambas as cultivares de batata-doce. Entretanto, este aumento não foi linear, atingindo-se produtividade máxima de $49,8 \mathrm{tha}^{-1} \mathrm{com}$ a aplicação de $325,5 \mathrm{~mm}$ para a 'Amanda' e 67,1 t ha-1 com a aplicação de 347,0 mm para 'Duda'. Estes valores representam a lâmina d'água a ser aplicada para a obtenção da máxima produtividade física para as cultivares (Figura 1). Ao estudar o efeito de diferentes doses de $\mathrm{N}, \mathrm{P}$ e K na produtividade das cultivares Amanda e Duda. Gonçalves (2012) obteve produtividades máximas de 40,5 e 47,2 $\mathrm{t} \mathrm{ha}^{-1}$, respectivamente, em condições de campo e sem irrigação. Andrade Júnior et al. (2009), trabalhando com as mesmas cultivares, encontraram produtividade que variou entre 22,0 e 45,4 t ha $^{-1}$ em cultivo sem irrigação, valores menores que os encontrados no presente trabalho. Cardoso et al. (2005) relataram produtividade superior a $20 \mathrm{t} \mathrm{ha}^{-1}$ para genótipos avaliados no campo, em Vitória da Conquista-BA. Zero \& Lima (2005) relatam que alcançaram produtividade de $18,2 \mathrm{t} \mathrm{ha}^{-1}$ em áreas onde foi feita rotação de cultura, estabelecendo uma correlação com o manejo do solo

Tabela 1. Características agronômicas da cultura da batata-doce utilizadas para a obtenção da evapotranspiração (agronomic characteristics of sweet potato crop used to calculate evapotranspiration). Viçosa, UFV, 2012.

\begin{tabular}{lccccc}
\hline Fase & $\begin{array}{c}\text { Duração } \\
\text { (dias) }\end{array}$ & ${ }^{1} \mathbf{K c}$ & ${ }^{2} \mathbf{Z}(\mathbf{c m})$ & ${ }^{3} \mathbf{P A S}(\%)$ & ${ }^{4} \boldsymbol{f}$ \\
\hline Pegamento & 20 & 0,50 & 0,1 & 2 & 0,5 \\
Sombreamento $<10 \%$ & 20 & 0,70 & 0,2 & 10 & 0,5 \\
Intermediário & 20 & 0,95 & 0,3 & 30 & 0,5 \\
Maximo $>80 \%$ & $110(136)^{5}$ & 1,25 & 0,3 & 100 & 0,5 \\
Final & 20 & 0,90 & 0,3 & 95 & 0,5 \\
\hline
\end{tabular}

${ }^{\mathrm{T}}$ Coeficiente cultural; ${ }^{2}$ profundidade efetiva do sistema radicular; ${ }^{3}$ area sombreada $(\%) ;{ }^{4}$ fator de disponibilidade hídrica; ${ }^{5}$ cultivar Duda. Os demais valores das variáveis são iguais para ambas as cultivares ( $\left({ }^{1} \mathrm{crop}\right.$ coefficient; ${ }^{2}$ root system depth; ${ }^{3}$ shaded area $(\%) ;{ }^{4}$ factor of water availability; ${ }^{5}$ Duda cultivar. The other variable values are equal for both cultivars). 


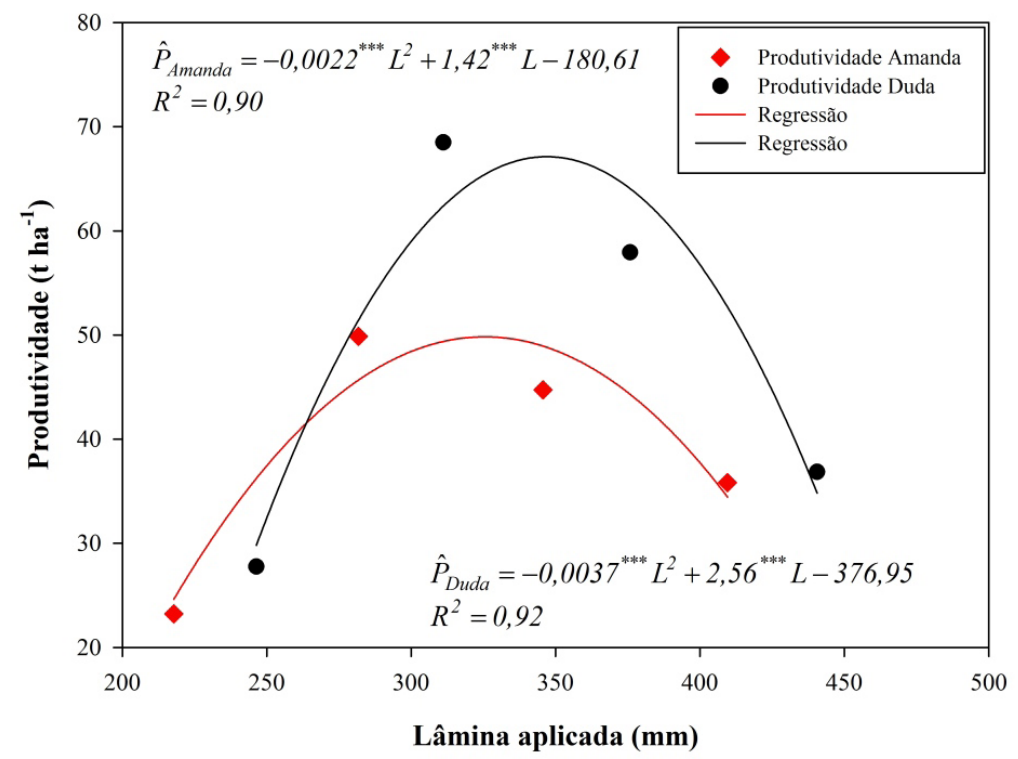

Figura 1. Produtividade de raízes tuberosas para cultivares Amanda e Duda ( $\mathrm{t}$ ha ${ }^{-1}$ ) em função da lâmina de irrigação aplicada no ciclo da cultura da batata-doce. ${ }^{* * *}$ significativo ao nível de $1 \%$ de probabilidade pelo teste "t" (productivity of tuberous roots of Amanda and Duda cultivars $\left(\mathrm{t} \mathrm{ha}^{-1}\right)$ depending on irrigation depth applied in the crop cycle for sweet potato; ***significant at 1\% probability by “t” test). Viçosa, UFV, 2012.

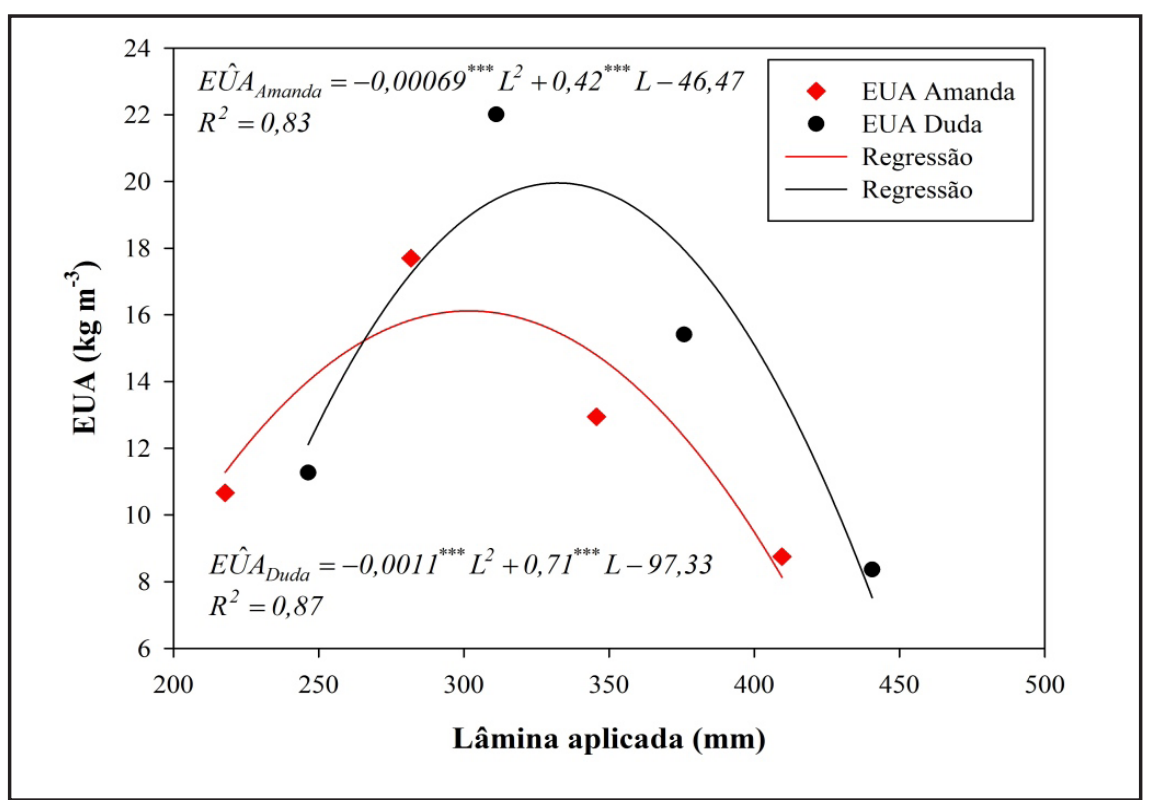

Figura 2. Uso eficiente da água $\left(\mathrm{kg} \mathrm{m}^{-3}\right)$ em função da lâmina de irrigação aplicada no ciclo da cultura da batata-doce para as cultivares Amanda e Duda. ${ }^{* * *}$ significativo ao nível de 1\% de probabilidade pelo teste " $\mathrm{t}$ " (water use efficiency $\left(\mathrm{kg} \mathrm{m}^{-3}\right)$ as a function of irrigation depth applied in the crop cycle for sweet potato of Amanda and Duda cultivars. ${ }^{* * *}$ significant at 1\% probability by “t”" test). Viçosa, UFV, 2012.

em Presidente Prudente-SP.

A produtividade de uma cultura depende de vários fatores, dentre eles, o climático, a radiação solar, a temperatura e o regime hídrico são extremamente importantes (Conceição et al., 2004). Se essas condições forem ideais, o potencial produtivo de uma cultura é maior quanto mais adaptado o genótipo for às condições de cultivo.
Bernardo (2006) observou para a cultura da cana-de-açúcar, que ao aumentar a quantidade de água aplicada, houve incremento positivo de produtividade até atingir um valor máximo. Após esse valor, houve decréscimo de rendimento com o aumento da lâmina total aplicada. Apesar do comportamento semelhante entre a cultura da cana-de-açúcar e a batata-doce, quanto ao fornecimento de água, deve-se destacar que a demanda hídrica da bata-doce é cerca de três vezes menor que a da cana-de-açúcar quando se compara a produtividade de etanol pelo volume de água consumida durante o ciclo das culturas (Dias, 2010). Esta comparação é de grande importância, pois reforça o potencial da batata-doce para a produção de etanol em áreas marginais onde o regime pluviométrico não permite $o$ cultivo da cana-de-açúcar.

Como a água é um fator limitante de produção, à medida que se aumenta sua disponibilidade, a cultura pode expressar melhor o seu potencial produtivo até um determinado ponto, após o qual a produtividade passa a decrescer, devido ao excesso de água no solo, à baixa aeração na zona radicular e à lixiviação de nutrientes (Bernardo, 2006), ou à ação de outro fator limitante, como a deficiência nutricional, por exemplo.

A maior eficiência no uso da água pela 'Duda' é evidenciada por sua maior produtividade em relação à 'Amanda', uma vez que esta maior produtividade $(20,7 \%)$ foi obtida com o aumento de apenas $6,2 \%$ na lâmina aplicada $(21,5$ $\mathrm{mm})$.

O aumento na lâmina de água aplicada resultou no aumento da eficiência do uso da água, até atingir um valor máximo de $16,1 \mathrm{~kg} \mathrm{~m}^{-3}$, com a aplicação de 301,8 mm para 'Amanda' e de 20,0 $\mathrm{kg} \mathrm{m}^{-3}$, com a aplicação de $332,4 \mathrm{~mm}$ para 'Duda'. Estes valores representam a lâmina de máxima utilização da água pelas cultivares de batata-doce estudadas (Figura 2). Os valores de EUA, obtidos por Gomes \& Carr (2003) com a cultivar TIS 2534 em Moçambique, ao longo dos períodos úmido e seco, foram de 1,3 e $2,4 \mathrm{~kg} \mathrm{~m}^{-3}$, respectivamente, e são muito inferiores aos observados para as cultivares Duda e Amanda no presente trabalho. 
Farias et al. (2008) estimaram a EUA em função da lâmina total aplicada (lâmina + precipitação efetiva) para a cultura da cana-de-açúcar, e observaram que os dados se ajustaram melhor ao modelo quadrático. $\mathrm{O}$ valor de máxima EUA observado foi de $7,2 \mathrm{~kg} \mathrm{~m}^{-3}$ para cana-de-açúcar irrigada com $100 \%$ da ETc e $4,0 \mathrm{~kg} \mathrm{~m}^{-3}$ para o cultivo sob regime de sequeiro. Resultados que reforçam a maior eficiência de utilização de água pela cultura da batata-doce em relação à cana-de-açúcar.

Com as lâminas de máximo uso da água pelas plantas de batata-doce e voltando à equação de produtividade em função da lâmina aplicada, foram obtidas as produtividades de 48,6 e 66,3 $\mathrm{t} \mathrm{ha}^{-1}$, referente às cultivares Amanda $\mathrm{e}$ Duda, respectivamente. Estes valores resultam em uma economia de água da ordem de 23,7 mm e uma redução de produtividade de apenas $1,2 \mathrm{t} \mathrm{ha}^{-1}$ para 'Amanda'. Para 'Duda' a economia de água seria de $14,6 \mathrm{~mm}$ e a redução na produtividade de $0,8 \mathrm{t} \mathrm{ha}^{-1}$.

Assim, pode-se concluir que a produtividade das cultivares de batata-doce foi expressivamente dependente da lâmina de água aplicada, sendo que as maiores produtividades foram alcançadas com a lâmina de 95,2 e 100,4\% da ETc, para 'Amanda' e 'Duda', respectivamente. A lâmina acumulada que proporcionou a maior eficiência no uso da água para as cultivares de batata-doce foram de 87,3 e 96,2\% da ETc, para 'Amanda' e 'Duda', respectivamente. A produtividade referente à máxima eficiência no uso da água para as cultivares de batatadoce foi obtida com economia de 237 e $146 \mathrm{~m}^{3} \mathrm{ha}^{-1}$ de água, para 'Amanda' e 'Duda', respectivamente.

\section{AGRADECIMENTOS}

Os autores agradecem ao Conselho Nacional de Desenvolvimento Científico e Tecnológico (CNPq) pela concessão de bolsa de estudo; à Fundação de Amparo à Pesquisa de Minas Gerais (FAPEMIG), pelo apoio financeiro, ao professor Márcio da Silveira, coordenador do programa de melhoramento da batata-doce da Universidade Federal do Tocantins por ceder as cultivares de batata-doce utilizada na pesquisa.

\section{REFERÊNCIAS}

ALLEN RG; PEREIRA LS; RAES D; SMITH M. 1998. Guidelines for computing crop water requeriments. Rome: FAO, 308 p. (FAO Irrigation and Drainage, 56).

ALVAREZ VVH; NOVAIS RF; DIAS LE; OLIVEIRA JA. 2000. Determinação e uso do fósforo remanescente. Viçosa: Sociedade Brasileira de Ciência do Solo. 25: 27-33 (Boletim Informativo).

ANDRADE JÚNIOR VC; VIANA DJS; FERNANDES JSC; FIGUEIREDO JA; NUNES UR; NEIVA PI. 2009. Selection of sweet potato clones for the region Alto Vale do Jequitinhonha. Horticultura Brasileira 27: 389-392.

BERNARDO S. 2006. Manejo da irrigação na cana-de-açúcar, Alcoolbrás. São Paulo, p. 72-80.

BERNARDO S; SOARES AA; MANTOVANI EC. 2006. Manual de irrigação. 8. ed. Viçosa, MG: Editora UFV. 625 p.

BUZETTI S; HERNANDEZ FBT; SA ME; SUZUKI MA. 1993. Influência da adubação nitrogenada e potássica na eficiência do uso da água e na qualidade de frutos de melão. Scientia Agrícola 50: 419-426.

CARDOSO AD; VIANA AES; RAMOS PAS; MATSUMOTO SN; AMARAL CLF; SEDIYAMA T; MORAIS OM. 2005. Avaliação de clones de batata-doce em Vitória da Conquista. Horticultura Brasileira 23: 911-914.

CONCEIÇÃO MK; LOPES NF; FORTES GRL. 2004. Partição de matéria seca entre órgãos de batata-doce (Ipomoea batatas (L.) Lam), cultivares Abóbora e da Costa. Revista Brasileira de Agrociência 10: 313-316.

DIAS LE. 2010. Culturas bioenergéticas para a produção de etanol. Boletim Informativo da Sociedade Brasileira de Ciência do Solo, Viçosa-MG, p. 10-15.

DINAR A. 1993. Economic factors and opportunities as determinants of water use efficiency in agriculture. Irrigation Science 14: 47-52.

DOORENBOS J; KASSAM AH. 1979. Yield response to water. Rome: FAO, 193 p. (FAO, Irrigation and Drainage Paper, 33).

DOORENBOS J; PRUITT JO. 1977. Guidelines for predicting crop water requirements. Rome: FAO, p 179. (FAO Irrigation and Drainage, 24).

EMPRESA BRASILEIRA DE PESQUISA AGROPECUÁRIA - EMBRAPA. 1997. Manual de métodos de análises de solo. 2.ed. Rio de Janeiro, 214p.

FARIAS CHA; FERNANDES PD; DANTAS NETO JY; GHEYI HR. 2008. Eficiência no uso da água na cana-de-açúcar sob diferentes lâminas de irrigação e níveis de zinco no litoral paraibano. Engenharia Agrícola 28: 494-506.

GONCALVES RC. 2012. Produtividade e absorção de nutrientes de três cultivares de batata-doce [Ipomoea batatas (L.) Lam] selecionadas para a produção de etanol, em função de diferentes doses de N, P e $\mathrm{K}$ aplicados no solo. Palmas: UFT. 66p. (Dissertação mestrado).

GOMES F; CARR MKV. 2003. Effects of water availability and vine harvesting frequency on the productivity of sweet potato in Southern Mozambique. II. Crop water use. Experimental Agriculture 39: 9-54.

IRRIPLUS. 2008. Sistema para Manejo de
Irrigação, Versão 2.8: UFV/GESAI/CIENTEC - Viçosa.

JALOTA SK; SOOD AGBS; CHAHAL BU. 2006. Crop water productivity of cotton (Gossypium hirsutum L.) - wheat (Triticum aestivum L.) system as influenced by deficit irrigation, soil texture and precipitation. Agricultural Water Management 84: 137-146.

KARATAS BS; AKKUZU E; UNAL HB; ASIK S; AVCI M. 2009. Using satellite remote sensing to asses irrigation performance in water user associations in the Lower Gediz Basin, Turkey. Agricultural Water Management 96: 982-990.

KELLER J; BLIESNER RD. 1990. Sprinkle and trickle irrigation. New York: Avibook, 649 p.

LÓPEZ-MATA E; TARJUELLO JM; JUAN JA; BALLESTEROS R; DOMÍNGUEZ A. 2010. Effect of irrigation uniformity on the profitability of crops. Agricultural Water Management 98: 190-198.

MANTOVANI EC; COSTA LC. 1998. Manejo integrado das culturas e dos recursos hídricos. Workshop internacional sobre manejo integrado das culturas e dos recursos hídricos. Brasília: SRH/MMA-DEA-UFV, $154 \mathrm{p}$.

OLIVEIRAAP; MOURA MF; NOGUEIRA DH; CHAVES NG; BRAZ MSS; OLIVEIRA MRT; BARBOSA JA. 2006. Produção de raízes de batata-doce em função do uso de doses de $\mathrm{N}$ aplicadas no solo e via foliar. Horticultura Brasileira 24: 279-283.

OLIVEIRA AP; OLIVEIRA MRT; BARBOSA JA; SILVA GG; NOGUEIRA DH; MOURA MF; BRAZ MSS. 2005. Rendimento e qualidade de raízes de batata-doce adubada com níveis de uréia. Horticultura Brasileira 23: 925-928.

PEREIRA LS; PAREDES P; EHOLPANKULOV ED; INCHENKOVA OP; TEODORO PR; HORST MG. 2009. Irrigation scheduling strategies for cotton to cope with water scarcity in the Fergana Valley, Central Asia. Agricultural Water Management 96: 723-735.

PEREIRA JÚNIOR LR; OLIVEIRA AP; GAMA JSN; CAMPOS VB; PRAZERES SS. 2008. Parcelamento do esterco bovino na produção de batata-doce. Revista Verde 3: 12-16.

SAEG. 2007. Sistema para análise estatísticas, Versão 9.1: Fundação Arthur Bernardes, UFV, Viçosa.

SANDRI D; MATSURA EE; TESTEZLAF R. 2007. Desenvolvimento da alface Elisa em diferentes sistemas de irrigação com água residuária. Engenharia Agrícola e Ambiental 11: 17-29.

SILVA JBC; LOPES CA; MAGALHÃES JS. 2008. Batata-doce: Ipomoea batatas. Brasília: EMBRAPA-CNPH. (Sistema de produção, n. 6).

SILVEIRA MA; ANDRÉ CMG; ALVIM TCl. 2007. A cultura da batata-doce como fonte de matéria-prima para produção de etanol. Palmas: UFT, 45 p. (Boletim Técnico).

SINGELS A; DONALDSON RA; SMIT MA. 2005. Improving biomass production and partitioning in sugarcane: theory and practice. Field Crops Research 92: 291-303.

YORDANOV I; VELIKOVA V; TSONEV T. 2003. Plant responses to drought and stress tolerance. Bulgarian Journal of Plant Physiology, Special Issue, p. 187-206.

ZERO VM; LIMA SL. 2005. Manejo e produtividade da cultura da batata-doce (Ipomoea batatas) no município Presidente Prudente-SP. Energia na Agricultura 20: 94-117. 